\title{
ANÁLISE DA PRODUÇÃO CIENTÍFICA EM ARTIGOS SOBRE ESTUDOS TRANSCULTURAIS NA AVALIAÇÃO PSICOLÓGICA EM BASES ONLINE
}

\author{
Maria Cristina Rodrigues Azevedo Joly ${ }^{1 *}$, Maria Inês Bustamante², Sandra Maria da Silva Sales Oliveira² \\ ${ }^{1}$ Universidade de Brasília, ${ }^{2}$ Universidade do Vale do Sapucaí
}

Recibido, abril 20/2013

Concepto de evaluación, noviembre 26/2013

Aceptado, diciembre 16/2014
Referencia: Joly, M. C. R. A., Bustamante, M. I., \& Oliveira, S. M. S. S. (2015). Análise da produção científica em artigos sobre estudos transculturais na avaliação psicológica em bases online. Acta Colombiana de Psicología, 18(1), 173-183. DOI: 10.14718/ACP.2015.18.1.16

Resumo

O avanço da Avaliação Psicológica enquanto especialidade indica a necessidade de estudos para analisar e caracterizar sua produção científica para o direcionamento de futuras investigações. Nesse sentido, analisaram-se as publicações nacionais e internacionais de estudos transculturais em Avaliação Psicológica, no período de 1992 a 2010 presentes em bases de dados eletrônicas. Os descritores pesquisados foram avaliação psicológica, psicometria, testes psicológicos e transcultural. Analisaram-se 239 resumos de artigos, dos quais 79 referiam-se à temática. O maior número de publicações encontra-se nas revistas Qualis B2 da Psicologia, constituindo-se em 8\% do total das publicações analisadas. A maioria dos estudos era de autoria de homens e mulheres e os anos de 2006 a 2010 apresentaram maior número de publicações. Quanto à metodologia de investigação, predominou a pesquisa de campo. É necessário a normatização da publicação dos resumos de artigos científicos que, em sua maioria, possuem informações incompletas, o que dificulta sua recuperação na íntegra e inviabiliza uma análise completa da produção da área.

Palavras-chave: produção científica; metaciência; cientometria; psicometria; estudos transculturais

\section{ANÁLISIS DE LA PRODUCCIÓN CIENTÍFICA DE ESTUDIOS TRANSCULTURALES EN EVALUACIÓN PSICOLÓGICA ENCONTRADOS EN BASES DE DATOS EN LÍNEA}

\begin{abstract}
El avance de la Evaluación Psicológica como especialidad indica la necesidad de estudios para analizar y caracterizar su producción científica con miras al direccionamiento de futuras investigaciones, Siguiendo dichos lineamientos, este trabajo analizó las publicaciones nacionales e internacionales de estudios transculturales en Evaluación Psicológica durante el período comprendido entre 1992 y 2010, presentes en bases de datos electrónicas. Los descriptores buscados fueron: evaluación psicológica, psicometría, pruebas psicológicas y transcultural. Se analizaron 239 resúmenes de artículos, de los cuales 79 se referían a la temática. El mayor número de publicaciones se encuentra en las revistas evaluadas por el Sistema Qualis B2 de Psicología, constituyéndose en el $8 \%$ del total de las publicaciones analizadas. La mayoría de los estudios fue escrita por hombres y mujeres, y entre los años 2006 y 2010 se presentó el mayor número de publicaciones. Con respecto a la metodología de investigación, predominó la investigación de campo. Es necesaria la normalización de la publicación de los resúmenes de artículos científicos que, en su mayoría, tienen información incompleta, lo cual dificulta su total recuperación e imposibilita un análisis completo de la producción en el área.

Palabras clave: producción científica, psicometría, cienciometría, estudios transculturales
\end{abstract}

\footnotetext{
* Doutora em Psicologia da Aprendizagem e Desenvolvimento Humano; Pesquisadora associada sênior no Programa de Pós-Graduação Stricto Sensu em Desenvolvimento Humano e Educação da Universidade de Brasília, DF, Brasil. Contato: Rua Dona Libânea, $2191 / 22$ 13015.090 Campinas, SP- Brasil. (+5519) 3233-4233. joly@unb.br
} 


\title{
ANALYSIS OF SCIENTIFIC PRODUCTION OF TRANSCULTURAL STUDIES ON PSYCHOLOGICAL ASSESSMENT FOUND ON ONLINE DATABASES
}

Abstract

\begin{abstract}
Advances in Psychological Assessment, as a specialization indicates the need for studies to analyze and characterize their scientific production in order to guide future research. Along these lines, this study examined the national and international publications of transcultural studies on Psychological Assessment from 1992 to 2010 found in electronic databases. The descriptors to be searched were: psychological assessment, psychometrics, psychological and transcultural testing. 239 abstracts of articles were analyzed, of which 79 were related to the research topic. The highest number of publications was found in the B2 Qualis Psychology System making up 8\% of the total publications analyzed..Most of the papers were written by both men and women, and between 2006 and 2010 the highest number of publications took place. The field research was the most common method used by the authors. It is necessary to standardize the publication of abstracts of scientific papers since most of them have incomplete information, making it difficult to achieve a full recovery and prevents the possibility of a thorough analysis of the production in this area.

Key words: Scientific production, psychometrics, scientometrics, transcultural studies
\end{abstract}

\section{INTRODUÇÃO}

A história da avaliação psicológica em nosso país se apresenta desde o início do século XX e se funde com a história da Psicologia. As décadas de 1960 e 1970 trouxeram um ponto desfavorável no que se refere a qualidade científica de seus artigos e livros, pois além de uma produção escassa no período, esta não atendia os princípios internacionais que norteavam a avaliação psicológica. No entanto, Noronha e cols. (2003) salientam um amadurecimento a partir de 1980, principalmente no que se refere a construção de instrumentos. Neste sentido, a década é tomada como referência, identificando alguns elementos e pessoas que marcaram a área. Por suposto, não se descartam contribuições para a avaliação psicológica antes desta época e, de fato, livros importantes de então continuam a ser citados (Baquero, 1974; Van Kolck, 1974).

Foi a partir da segunda metade da década de 1990 que surgiram alguns dos primeiros manuais práticos de elaboração de testes e escalas (Pasquali, 1997, 1999). Ainda nesse mesmo período, no ano de 1997, foi criado o Instituto Brasileiro de Avaliação Psicológica, que em 2002 lançou a revista Avaliação Psicológica, editada pela Casa do Psicólogo. Juntamente com os laboratórios de avaliação e medida em Psicologia, que por volta dos anos 1990 começaram a se consolidar em diversas universidades brasileiras (Universidade Federal do Rio Grande do Sul, Universidade de Brasília, Universidade Estadual de Campinas e Universidade do Vale do Rio dos Sinos). Estes eventos impulsionaram a produção de pesquisa e publicação brasileira na área.

Soma-se a isto, a resolução n0 25/2001 (Conselho Federal de Psicologia, 2001) que estabelece os critérios científicos mínimos, a constituição de uma comissão de especialistas (Comissão Consultiva) na área para análise dos testes, a criação de cursos de Pós-Graduação Stricto Sensu (Universidade São Francisco-Itatiba/SP) contribuindo para formação de especialista na área. Estes, dentre outros eventos, podem ser citados como alguns dos esforços no sentido de fortalecimento da área da avaliação psicológica e principalmente na melhoria da qualidade dos instrumentos psicológicos.

Outras contribuições importantes, não restritas à área de avaliação psicológica, mas dignas de menção para seu desenvolvimento, foram os fomentos à pesquisa, com Editais Universais e de Ciências Humanas e Sociais, o aumento das bolsas PQ (Produtividade em Pesquisa) e incentivo ao pós-doutorado, e o portal periódicos da Coordenação de Aperfeiçoamento de Pessoal de Nível Superior (CAPES). Revistas de escasso acesso, como Educational and Psychological Measurement, Psychological Assessment e Personality and Individual Differences se tornaram mais correntes nas universidades, com disponibilidade para os estudantes e pesquisadores (Gouveia, 2009).

Ao lado disso, para Oliveira, Noronha, Dantas e Santarém (2005) a avaliação pode fornecer elementos de análise imprescindíveis para a atuação do psicólogo em diferentes campos da psicologia. Isso não quer dizer que não se tenha problemas. Os estudos realizados por Noronha, Sartori, Freitas e Ottati (2001) em análise a 21 instrumentos de inteligência sobre informações relativas aos dados de identificação e de aplicação indicam que nos manuais dos testes, por exemplo, verificou-se ausência de informações como data de publicação, nome do autor ou bibliografia.

Em outra pesquisa realizada com 214 psicólogos da região 06, que compreende São Paulo com suas subsedes em Assis, Baixada Santista, Bauru, Campinas, Grande ABC, 
Ribeirão Preto, São José do Rio Preto e Vale do Paraíba, Noronha (2002) obteve como parte dos resultados, que os psicólogos esperavam que fossem lançados no mercado novos instrumentos com diferentes tipos de abordagens e construtos, porém, com padronização e normatização para a realidade brasileira; que estes instrumentos apresentem estudos de validade e precisão; que os manuais sejam mais completos e atendam a diferentes realidades sócio-culturais e que os custos dos materiais não sejam tão altos. Noronha e cols. (2003), corroborando dados da pesquisa anterior, alertam para fato de que não basta apenas um instrumento apresentar padronização e normas, é preciso fundamentalmente verificar a validade sob diferentes perspectivas, focando no construto, no conteúdo e no critério.

Fica claro que a psicologia e a avaliação psicológica devam procurar formas mais apuradas de observação dos seus fenômenos. Em específico pode-se dar destaque às escalas, que têm demonstrado bastante eficácia na mensuração de dimensões psicológicas e por suas características de sistematização do objeto pesquisado. Andrade e Gorenstein (1998) e Nardi (1998) concordam que a utilização de escalas garante que a informação coletada sobre sintomas específicos seja padronizada e passível de comparação de forma mais confiável e que os escores obtidos representem um padrão sintomatológico consistente e comum ao maior número de pacientes com o transtorno medido.

Primi (2010) apresenta um panorama de questões importantes da área de avaliação psicológica nos últimos 25 anos no Brasil. Discute fundamentos epistemológicos da área, bem como suas relações com a ciência e a integração do pensamento nomotético e idiográfico da pesquisa com a prática profissional. Faz um apanhado histórico de eventos importantes e uma análise geral da produção de artigos. Descreve-se também a produção de instrumentos e o Sistema de Avaliação de Testes Psicológicos do Conselho Federal de Psicologia. Por fim, aponta perspectivas para o futuro em quatro áreas: avanços metodológicos e tecnológicos, integração de abordagens e avanço dos seus métodos, validade consequencial e relevância social, e incentivo à formação e à criação da especialidade em avaliação psicológica.

Determinados setores da psicologia tem se movimentado em prol da avaliação psicológica por meio de congressos, publicações em revistas científicas, cursos específicos de pós-graduação entre outros. Autores como Anastasi e Urbina (2000); Sisto (2004); Joly e Noronha (2006); Urbina (2007); Noronha e Baptista (2007) dentre outros, têm enfatizado a necessidade de se estar atentos aos parâmetros psicométricos, principalmente estudos de validade e precisão, ao se construir testes psicológicos bem como seguir rigorosamente todos os critérios desde o princípio da tarefa.
No que se refere a adaptação de instrumentos psicológicos, Argollo e cols. (2009) esclarecem que este processo têm sido amplamente utilizado em pesquisas latino-americanas como meio para o desenvolvimento de novos recursos adequados de diagnóstico de desempenho cognitivo para o contexto nacional. Este processo é extremamente rico, complexo e possibilita uma avaliação dos construtos psicológicos cada vez mais acurada e contextualizada, além de possibilitar o intercâmbio de informações entre a comunidade científica internacional com, por exemplo, estudos transculturais.

Para que a adaptação transcultural seja possível, é necessário que a forma de mensuração e interpretação do teste original seja adequada à natureza do grupo alvo para que não existam vieses que restrinjam a validade e a fidedignidade do mesmo na transição de seu uso do país de origem para o que será adaptado. Anastasi e Urbina (2000) ressaltam a importância disto afirmando que pessoas com backgrounds distintos podem utilizar estratégias diferentes para resolver um mesmo problema de um teste.

Segundo Mattos e cols. (2006), a tradução e a adaptação transcultural de instrumentos diagnósticos ou escalas de avaliação deve seguir o paradigma "emic-etic". O vértice "emic" (termo retirado da palavra phonemic) diz respeito a um olhar do fenômeno a partir do próprio contexto ou da cultura em que ele ocorre; já o vértice "etic" (termo obtido a partir da palavra phonetic) diz respeito a uma generalização do fenômeno observado para comparação em diferentes culturas. Dentro dessa perspectiva, a tradução de uma escala requer cuidados lingüísticos, uma vez que termos podem ter diferentes abrangências e especificidades inerentes a cada idioma. Além disso, a validação semântica é necessária para a certificação de que o instrumento é compreensível a todos os membros da população à qual se destina (Pasquali, 1998).

Diante da escassez de estudos transculturais em geral e especificamente no que se refere ao levantamento feito, não se identificaram na análise da literatura, a realização de análises da produção de estudos transculturais para Avaliação Psicológica. Foi possível verificar estudos dessa natureza existentes para outras áreas da Psicologia como a Psicologia Social (Ribas Junior \& cols., 2009) e a Psicologia Geral (Maluf, 2004). Considerando, como descrito anteriormente, o avanço da área, faz-se necessário avaliar a produção específica da Avaliação Psicológica e os estudos transculturais com a contribuição da metaciência.

A metaciência tem como perspectiva analisar a produção científica de uma área de conhecimento (a partir de seu volume de publicações, saliência e qualidade) é uma tarefa importante e necessária para o mapeamento das forças e fraquezas da mesma e para o direcionamento 
de futuras investigações (Bufrem \& Prates, 2005; Witter, 1999). Segundo Witter (1999) as pesquisas em metaciência, que são investigações que avaliam pesquisas, possibilitam verificar a qualidade do conhecimento produzido em uma determinada área, suas necessidades e déficits a partir de dimensões mais abrangentes e pontuais. A análise pode ser feita levando-se em consideração a temática, o discurso, o enfoque teórico, a metodologia, o procedimento de análise de dados.

Em acréscimo, Lopez Lopez, Silva, Garcia-Cepedro, Aguilar-Bustamante e Lopez (2010) assinalam a relevância de estudos cientométricos especialmente para dar visibilidade à produção na America Latina e, assim, possibilitar a organização de redes de pesquisadores e estudos transculturais na Psicologia. Nesse sentido, este trabalho buscou realizar uma análise das publicações nacionais e internacionais de artigos sobre Avaliação Psicológica para mapear as produções na área de estudos transculturais.

\section{MÉTODO}

\section{Amostra}

A busca pelas produções científicas foi realizada em setembro e outubro de 2010, nas bases de dados indicadas anteriormente. Foram identificados 239 artigos referentes ao estudo com os descritores, sendo 104 encontrados na base BVS-PSI, 41 na LILACS, 4 na Indexpsi, 8 na Redalyc e 82 na Scielo. Dos 239 artigos 14 se repetiam nas diferentes bases de dados, todos pertinentes ao estudo e só foram inseridos em sua primeira aparição. Após leitura e seleção dos resumos, no que se refere a pertinência do estudo, chegou-se a 79 registros que foram submetidos à análise.

\section{Instrumento}

Foram utilizados como fonte de análise os periódicos indexados da base de dados Scielo, Lilacs, Redalyc, BVS-PSI e Indexpsi. Estas têm como objetivo garantir o acesso à informação de forma rápida e precisa, pela seleção, organização e disseminação destas informações num espaço virtual informatizado. Assim, garante ao psicólogo e ao pesquisador da psicologia o acesso eficiente, on-line, a publicações científicas. Como instrumento de pesquisa busca atualizar o profissional da área assim como, permitir o avanço da pesquisa cientifica e são modelos que proporcionam soluções eficientes para assegurar a visibilidade e o acesso universal à literatura científica.

\section{Procedimento}

A partir do resultado da busca nas bases de dados, foi feita uma leitura rigorosa de cada um dos resumos para determinar quais pertenciam ao objetivo desse estudo. Os dados foram catalogados em uma planilha composta pelas categorias de análise a serem consideradas para a seleção dos resumos. As categorias selecionadas basearam-se em alguns critérios da metaciência definidos por Witter (1999) como a temática, autoria, discurso e o método.

No tocante aos critérios definidos para análise pela metaciência, a categoria temática considerou a quantidade de resumos sobre avaliação psicológica, a área de investigação e o tema principal abordado pelo artigo publicado. Estes critérios foram analisados por meio do título, do conceito exposto no resumo e pelas palavras-chave. Para a análise do tipo da autoria observou-se se o resumo era de autoria feminina, masculina ou mista. Para o discurso, identificouse o tipo de artigo (artigo teórico, de pesquisa, revisão de literatura ou estudo de caso) considerando-se para os artigos de pesquisa a presença ou ausência de objetivos, resultados e discussão; para os artigos teóricos a presença ou ausência de apresentação da temática, desenvolvimento e conclusão. No quesito método avaliou-se a presença ou ausência de participantes, materiais, procedimentos, o tipo de pesquisa (levantamento, experimental, quase-experimental ou construção de instrumento) e a análise de dados (quantitativa, qualitativa ou mista). Além dessas categorias, foi realizada uma análise do perfil da revista, a área de conhecimento a que se destina e a classificação Qualis.

\section{RESULTADOS}

Os resultados foram analisados por meio da estatística descritiva buscando apresentar a caracterização das variáveis, perfil das revistas e os critérios da metaciência (temática, autoria, discurso, metodologia e análise de dados). Utilizou-se a Prova Qui-quadrado para verificar possíveis diferenças entre os resultados observados e os esperados no tocante à produção científica para as variáveis consideradas individualmente e associadas ao período de análise das revistas (1992-2010).

Os 79 artigos analisados encontravam-se distribuídos em revistas científicas, classificadas no Qualis-Periódicos CAPES. Os dados apresentados na tabela 1 mostram que a maioria das produções foram publicadas em revistas de classificação B2, com uma frequência de 22 resumos, o que equivale a $27,8 \%$ do total das publicações analisadas; a categoria B5 contou com menor número de publicações, num percentual de $1,3 \%$. 
Tabela 1.

Distribuição de frequência e porcentagem da classificação Qualis das revistas em que foram publicados os resumos analisados

\begin{tabular}{ccc}
\hline Classificação Qualis & Frequência & Porcentagem \\
\hline A1 & 16 & 20,3 \\
\hline A2 & 12 & 15,2 \\
\hline B1 & 8 & 10,1 \\
\hline B2 & 22 & 27,8 \\
\hline B3 & 3 & 3,8 \\
\hline B4 & 17 & 21,5 \\
\hline B5 & 1 & 1,3 \\
\hline Total & 79 & 100,0 \\
\hline
\end{tabular}

Quanto aos indexadores, a base Scielo foi a que apresentou o maior número de resumos $(41,8 \%)$ e o menor número foi encontrado na base Redalyc (5,1\%). Atualmente o Scielo é um importantíssimo meio eletrônico da divulgação da produção nacional, recurso bastante utilizado por agregar parte significativa da produção científica brasileira. A base Indexpsi foi a que apresentou menor quantidade de publicações ( $\mathrm{n}=3 ; 3,8 \%)$. Vale esclarecer que a BVS-PSI Brasil, considerando a etapa de levantamento das produções, foi a que teve o maior número de publicações na área, com um total de 104 artigos. Entretanto, a maioria não apresentava resumo e/ou não se referia ao objeto de estudo desta investigação, apenas 10 resumos foram considerados pertinentes ao tema em estudo, e, ainda assim, dois deles já se encontravam em outras bases de dados.

Tabela 2.

Distribuição de frequência e porcentagem das bases de dados em que foram publicados os resumos analisados

\begin{tabular}{ccc}
\hline $\begin{array}{c}\text { Bases } \\
\text { de Dados }\end{array}$ & Frequência & Porcentagem \\
\hline BVS-Psi & 11 & 13,9 \\
\hline Lilacs & 28 & 35,4 \\
\hline Indexpsi & 3 & 3,8 \\
\hline Redalyc & 4 & 5,1 \\
\hline Scielo & 33 & 41,8 \\
\hline Total & 79 & 100,0 \\
\hline
\end{tabular}

Considerando a quantidade de publicações no período de 1992 a 2010, o ano de 2008 é o que possui o maior número de publicações na área (21,5\%) e o período de 1992 a 1999 o menor (1,3\%) por ano. Percebe-se ainda, que a frequência das publicações oscilam ao longo dos anos, entretanto, deve-se ressaltar que ocorre um aumento de publicações nos últimos anos, o que confirma o esperado para a produção científica de uma área, que com o passar dos anos haja um crescimento sobre o conhecimento adquirido na mesma (Tabela 3).

Tabela 3

Distribuição de frequência e porcentagem do ano de publicação dos resumos analisados

\begin{tabular}{ccc}
\hline \multirow{2}{*}{ Ano de publicação } & \multicolumn{2}{c}{ Número de resumos } \\
\cline { 2 - 3 } & Frequência & Porcentagem \\
\hline $1992-1999$ & 5 & 6,5 \\
\hline $2000-2005$ & 27 & 34,2 \\
\hline $2006-2010$ & 47 & 59,3 \\
\hline Total & 79 & 100,0 \\
\hline
\end{tabular}

Focalizando como primeiro critério de análise da metaciência, a temática, procedeu-se a organização dos resumos por área específica, classificando-os, portanto em quatro grupos, a saber, aspectos psicológicos, transtornos mentais, saúde física e qualidade de vida.

A seguir, na tabela 4, pode-se observar a frequência e a porcentagem considerando a área de investigação específica de cada resumo. Os resultados não demonstraram equivalência entre a quantidade das produções científicas publicadas nos grupos, no entanto, a área que se refere a aspectos psicológicos apresenta o maior número de artigos e o menor, saúde física.

Tabela 4.

Distribuição de frequência e porcentagem por área de investigação específica dos resumos

\begin{tabular}{ccc}
\hline $\begin{array}{c}\text { Área de } \\
\text { investigação }\end{array}$ & Frequência & Porcentagem \\
\hline aspectos psicológicos & 41 & 51,9 \\
\hline transtornos mentais & 19 & 24,1 \\
\hline saúde física & 9 & 11,4 \\
\hline qualidade de vida & 10 & 12,7 \\
\hline Total & 79 & 100,0 \\
\hline
\end{tabular}

A tabela 4 permite identificar que os estudos foram realizados, em sua maioria, na área da psicologia, fato este que já era esperado considerando o objeto de estudo do presente artigo. Embora a maioria de trabalhos se refira a aspectos psicológicos, ainda são poucos os estudos realizados, o que demonstra a necessidade de mais investigações sobre a área.

Foi verificado também em cada resumo, qual foi o tema principal abordado pelo pesquisador. Isto porque esse item 
permite observar para qual informação os pesquisadores estão dando mais atenção, bem como as tendências e as necessidades de uma determinada área, afim de possibilitar estudos que aprimorem o conhecimento disponível (Noronha \& cols., 2006; Pacheco, 2005; Witter, 1999).

A tabela 5 mostra a identificação dos objetos de investigação dos temas catalogados a partir dos conceitos que mais apareceram nas produções analisadas. Constatou-se que os testes foram os que apresentaram o maior número de relato de investigações 74 (93\%).

\section{Tabela 5.}

Distribuição de frequência e porcentagem por objetos de investigação dos temas catalogados

\begin{tabular}{ccc}
\hline Objetos de & \multicolumn{2}{c}{ Número de artigos } \\
\cline { 2 - 3 } investigação & Frequência & Porcentagem \\
\hline teste & 74 & 93,7 \\
\hline informática & 1 & 1,3 \\
\hline base de dados & 1 & 1,3 \\
\hline psicólogos & 2 & 2,5 \\
\hline entrevistas & 1 & 1,3 \\
\hline Total & 79 & 100,0 \\
\hline
\end{tabular}

No que se refere às áreas de investigação dos artigos estudados referentes a avaliação psicológica e estudos transculturais, estes puderam ser classificados em: psicologia, odontologia, medicina, fisioterapia e saúde publica (ver Tabela 6). Observa-se que a maioria dos artigos referem-se a Psicologia seguida pela Medicina, a Odontologia e a Fisioterapia que contaram com menor frequência de resumos analisados.

Tabela 6.

Distribuição de frequência e porcentagem por área de investigação

\begin{tabular}{ccc}
\hline Área & Frequência & Porcentagem \\
\hline Psicologia & 47 & 59,5 \\
\hline Odontologia & 2 & 2,5 \\
\hline Medicina & 24 & 30,4 \\
\hline Fisioterapia & 1 & 1,3 \\
\hline Saúde Pública & 5 & 6,3 \\
\hline Total & 79 & 100,0
\end{tabular}

Torna-se relevante explicitar os critérios utilizados para as definições dos campos de investigação (Tabela 6) e também, a título de ilustração, referenciar algumas produções analisadas, de acordo com a classificação em cada categoria. Classificaram-se como categoria psicologia os artigos que abordaram a avaliação psicológica considerando a apresentação de suas definições e sua aplicação frente a cursos e instituições. Pode-se citar como exemplo o trabalho de Pesce e cols. (2005) que se refere a adaptação transcultural, confiabilidade e validade de uma escala de resiliência. A escala foi adaptada para uma amostra de escolares dos ensinos fundamental e médio da rede pública de ensino do Município de São Gonçalo, Rio de Janeiro, Brasil em que são apresentados dados do estudo-piloto, com 203 alunos entrevistados em dois momentos consecutivos (teste-reteste), bem como do total de alunos investigados na pesquisa (977). Quanto à adaptacão transcultural, foram encontrados bons resultados na equivalência semântica dos itens, tanto para o significado geral, quanto para o referencial. $\mathrm{O}$ alfa de Chronbach encontrado foi de 0,85 no pré-teste e 0,80 na amostra total.

Quanto a categoria odontologia pode-se citar o trabalho intitulado Equivalência semântica da versão em português do instrumento Early Childhood Oral Health Impact Scale de Tesch, Oliveira e Leão (2008) que contou com 20 participantes, com o objetivo de avaliar a equivalência semântica entre o ECOHIS e sua versão em português, instrumento que avalia a qualidade de vida relacionada a saúde bucal de crianças pré-escolares e seus familiares. A metodologia incluiu 6 etapas relativas a traduções, retraduções e revisões, o que permitiu obter uma versão brasileira que possui equivalência semântica com o instrumento original.

O estudo classificado no campo da fisioterapia realizado por Saliba, Magalhães, Faria, Laurentino, Cassiano e Teixeira-Samela (2011) e denominado "Adaptação transcultural e análise das propriedades psicométricas da versão brasileira do instrumento Motor Activity Log" foi do tipo exploratório e visou a adaptação do Mal para a população brasileira tendo com participantes indivíduos hepiplégicos. O MALé um instrumento comumente utilizado para avaliar funcionalmente a habilidade motora do membro superior mais utilizado após o indivíduo ter sofrido um acidente vascular encefácilo (AVE). Ao lado disso, investiga como se deu o aprendizado do "não uso" desse membro após o AVE em atividades da vida diária. Os autores tiveram como referencia o estudo de Saliba, Chaves Junior, Faria e Teixeira-Samela (2008) que descreveu as propriedades psicométricas já estabelecidas para a MAL e avaliou a sua potencialidade para ser utilizado na população brasileira. Os resultados das escalas quantitativa e qualitativa, analisados pelo modelo de Rasch, revelaram um CCI de 0,98. Apesar disso, foram identificados itens erráticos que indicaram discrepância entre dificuldade dos itens e habilidade da amostra. Os autores constataram a possibilidade de uso do MAL-Brasil com hemiplégicos crônicos sem compro- 
metimento severo, sendo necessárias outras pesquisas para busca de outras evidências de validade mais abrangentes do instrumento. esse estudo ratifica a necessidade de estudos de adaptação transcultural de instrumentos para o Brasil, independente dos mesmos já apresentaram qualidades psicoméricas em outros países nos quais á houve adaptação de sua forma original.

Dentre os estudos classificados na área medicina salienta-se o de Silva e Thuler (2008) denominado "Tradução e adaptação transcultural de duas escalas para avaliação da dor em crianças e adolescentes" cujo objetivo foi traduzir, retrotraduzir e adaptar culturalmente o conteúdo da Face, Legs, Activity, Cry, Consolability (FLACC) e da Escala de Faces Revisada (FPS-R) para a avaliação da dor em crianças escolares e adolescentes brasileiros. Foi realizada tradução da escala original do inglês para o português. As escalas obtidas foram retrotraduzidas e revisadas de maneira independente. A adaptação transcultural incluiu a submissão das versões revisadas das escalas a 12 experts visando melhorar a compreensão, correspondência e aceitação dos itens. Um pré-teste foi realizado em uma amostra de conveniência (20 pacientes e 22 profissionais de saúde) para avaliação do conteúdo das escalas. Os pacientes com câncer, entre 7 e 17 anos de idade, encontravam-se em acompanhamento no ambulatório ou na enfermaria de pediatria do Instituto Nacional de Câncer. Após a incorporação das recomendações dos diferentes profissionais participantes do processo de tradução, retrotradução e avaliação de conteúdo das escalas, o pré-teste mostrou que 90 e 100 por cento, respectivamente, compreenderam o conteúdo das escalas, tendo a pontuação média para compreensão variado entre 8,8 e 10,0, em uma escala de 0 a 10 na qual os escores mais elevados indicam melhor compreensão. Ambas as escalas se mostraram de fácil compreensão para avaliação da dor em crianças e adolescentes brasileiros com câncer.

No que concerne à saúde pública pode-se citar o estudo de Paixao Jr, Reichenheim, Moraes, Coutinho e Veras (2007) denominado "Adaptação transcultural para o Brasil do instrumento Caregiver Abuse Screen (CASE) para detecção de violência de cuidadores contra idosos". O CASE foi originalmente desenvolvido no Canadá e utilizado para rastrear violências em idosos entrevistando seus cuidadores. $\mathrm{O}$ processo de avaliação de equivalências conceitual e de itens, que envolveu uma ampla e sistemática revisão bibliográfica, consistiu de uma discussão em grupo de expertos. A equivalência semântica envolveu duas traduções e respectivas retraduções em paralelo; uma avaliação de equivalência de significados referencial e geral entre o CASE original e as versões em português; discussões posteriores com o grupo de expertos para definir a versão final; e um pré-teste com quarenta cuidadores de pacientes idosos em um serviço de atendimento ambulatorial de geriatria. Foi possível estabelecer uma versão em português para o Brasil com boa qualidade de equivalência conceitual, de itens e semântica.

Considerando a importância de analisar a qualidade da informação disponível nos resumos publicados nas bases de dados em pauta, verificou-se, tendo por referência a metaciência, a presença ou ausência de objetivos, resultados e discussão dos artigos. Observa-se que apenas três não apresentam objetivos, dois não apresentam resultados e 31 não apresentam discussão (Tabela 7).

Tabela 7.

Distribuição de frequência e porcentagem referente a presença e ausência de objetivo, resultado e discussão nos artigos

\begin{tabular}{cccc}
\hline & Objetivo & Resultado & Discussão \\
\hline ausência & 3 & 2 & 31 \\
\hline presença & 76 & 77 & 48 \\
\hline Total & 79 & 79 & 79 \\
\hline
\end{tabular}

Quanto a descrição do número de palavras-chave apresentadas nos artigos não se observa um número uniforme destas citadas nos artigos. Há artigos com duas, três, quatro e até dez palavras-chave. A frequência maior de ocorrência de palavras-chave encontra-se entre três e cinco, sendo que com três há 29 artigos, com quatro 17 e com cinco 18. Estes resultados vão ao encontro com as normas de publicação que revistas científicas estabelecem para número de palavras-chave.

Ao analisar os autores dos resumos, foram encontrados três artigos sem os mesmos, ou seja, eles traziam apenas um autor e et al., não deixando a possibilidade de identificar o tipo de autoria. Pode-se inferir que a maioria dos artigos é de autoria mista e os de autoria masculina são os que apresentam menor ocorrência.

Visando verificar o tipo de artigo, se de pesquisa de campo ou teórico, procedeu-se a análise considerando que dos 79 artigos avaliados 72 referem-se a pesquisa de campo e apenas 7 artigos são teóricos e quanto os resultados, observou-se que apareceram descritos na maioria das publicações $(\mathrm{N}=79)$. Por sua vez, a metodologia foi descrita em 71 resumos de pesquisa de campo, bem como procedimentos em 65 resumos.

Os resultados do Teste Qui-quadrado para um critério demonstraram diferenças estatisticamente significativas para base de dados pesquisadas $\left(\chi^{2}[4]=48,785 ; p<0,001\right)$, Qualis da revista $\left(\chi^{2}[6]=31,494 ; p<0,001\right)$, ano de publicação $\left(\chi^{2}[15]=71,481 ; p<0,001\right)$ objeto de investigação 
$\left(\chi^{2}[4]=268,025 ; p<0,001\right)$,área de investigação $\left(\chi^{2}[4]=99,165\right.$; $\mathrm{p}<), 001)$, tipo de artigo $\left(\chi^{2}[1]=53,481 ; \mathrm{p}<0,001\right)$, tipo de análise de dados - quantitativa ou qualitativa $\left(\chi^{2}[1]=43,556\right.$; $\mathrm{p}<0,001)$, tipo de pesquisa - levantamento, construção de instrumento, evidências de validade $\left(\chi^{2}[2]=74,846 ; p<0,001\right)$ e elementos do discurso presentes nos artigos e considerados como os objetivos $\left(\chi^{2}[1]=66,462 ; \mathrm{p}<0,001\right)$, participantes $\left(\chi^{2}[1]=28,595 ; \mathrm{p}<0,001\right)$, materiais $\left(\chi^{2}[1]=57,316 ; \mathrm{p}<0,001\right)$, procedimentos $\left(\chi^{2}[1]=44,507 ; \mathrm{p}<0,001\right)$, resulta$\operatorname{dos}\left(\chi^{2}[1]=70,205 ; p<0,001\right)$, número de palavras-chave $\left(\chi^{2}[7]=75,430 ; p<0,001\right)$ e tipo de autoria $\left(\chi^{2}[2]=34,132\right.$; $\mathrm{p}<0,001)$. Não se verificou diferença estatisticamente significativa para discussão $\left(\chi^{2}[1]=3,282 ; p<0,070\right)$.

Considerando dois critérios para análise, identificou-se diferença estatisticamente significativa para área de investigação e base de dados $\left(\chi^{2}[60]=85,764 ; p=0,05\right)$, ano de publicação em relação ao Qualis da revista $\left(\chi^{2}[60]=152,761\right.$; $\mathrm{p}<0,001)$ e base de dados pesquisada $\left(\chi^{2}[16]=25,998\right.$; $\mathrm{p}=0,016)$. Não se constataram diferenças estatisticamente significativas para ano de publicação em relação à área de investigação $\left(\chi^{2}[60]=38,054 ; p=0,99\right)$ e temática $\left(\chi^{2}[45]=58,558 ; \mathrm{p}=0,085\right)$.

\section{DISCUSSÃO}

À guisa de síntese, constatou-se na análise da produção científica de estudos transculturais em Avaliação Psicológica, no período de 1992 a 2010, que dos 79 resumos analisados, $41,8 \%$ encontravam-se na base de dados Scielo. Distribuídas em revistas, a maioria das produções foram publicadas em revistas de classificação Qualis B2 (27,8\%). É importante destacar que as produções têm visibilidade especialmente no cenário nacional e latinoamericano por se encontrarem na Scielo, favorecendo assim, investigações que se complementem possibilitando, de fato, um avanço real da área (Witter, 2006; Lopez Lopez \& cols,. 2010; Pacheco, 2005). A despeito disso, salienta-se que cabe uma investigação futura, de caráter mais qualitativo, acerca dos motivos que levaram os autores a publicar, com mais frequência, suas pesquisas em revista B2. Era de se esperar, considerando Packer e Meneghini (2006), que as informações dos estudos em análise tivessem maior visibilidade e impacto científico e profissional, em função de quão inéditas elas são, e, também, de seu caráter internacional por se tratarem de estudos transculturais, daí sua publicação, hipoteticamente, deveria ocorrer em revistas A. Ao lado disso, poder-se-ia investigar com os editores das revistas Qualis A acerca da frequência de submissão desses estudos aprovados e reprovados e quais os motivos.

Em relação ao número de publicações, o período de 2006 a 2010 foi o que apresentou maior quantidade de artigos em Avaliação Psicológica (59,3\%). Tal resultado revela uma continuidade do processo de produção científica da área, colaborando com a geração de conhecimento científico para além da área específica em análise no presente estudo, de acordo com a perspectiva de Witter (1999; 2002) e Pacheco (2005). Cabe salientar que a área de Avaliação Psicológica encontra-se atualmente em expansão. Considerando-se a criação de cursos de especialização e pós-graduação; a organização de laboratórios de pesquisa os quais têm criado redes de pesquisadores; o Instituto Brasileiro de Avaliação Psicológica (IBAP), com a promoção de congressos e eventos que fomentam a produção científica e principalmente a resolução do Conselho Federal de Psicologia (2001) que estabelece critérios para uso de instrumentos e Conselho Federal de Psicologia, procedimentos avaliativos. Tal expansão implica em definições quanto ao caminho a ser percorrido de modo que as investigações realizadas ampliem o escopo científico da área já existente.

Torna-se importante uma reflexão e análise sobre quais são as reais contribuições da Avaliação Psicológica para a Psicologia, bem como uma análise de artigos internacionais e estudos transculturais encontrados nas revistas de Avaliação Psicológica. Com isto torna-se possível obter um panorama do que tem sido estudado e publicado fora do Brasil, como destacam Gouveia (2009) e Primi (2010). Sugere-se que haja investimento maior em pesquisas transculturais que possam revelar a organização e/ou fortalecimento de redes de investigação entre os países como indicam Lopez Lopez e cols., (2010).

A temática mais investigada foi aspectos psicológicos $(51,9 \%)$, referente a trabalhos que apresentaram a avaliação psicológica e validação de instrumentos a partir de suas definições e aplicação, o que pode indicar, por um lado, os avanços quanto a expansão da avaliação psicológica atualmente. Todos os artigos tiveram como enfoque teórico a psicometria. Este avanço se dá quer pela criação de cursos de especialização e pós-graduação stricto sensu, pela organização de laboratórios de pesquisa os quais tem criado rede de pesquisadores, quer pela resolução do Conselho Federal de Psicologia que estabeleceu critérios para o uso de instrumentos e procedimentos avaliativos (Joly \& Noronha, 2006).

Constatou-se ainda, que a maioria dos estudos realizados foi de autoria mista (62\%\%) seguido pela feminina $(21,5 \%)$. A este respeito Joly, Martins, Abreu, Souza e Cozza (2004) explicam que a pesquisa escrita em maior número mista $\mathrm{e}$ feminina se dá pelo fato de a maioria de profissionais na área serem do sexo feminino. Quanto ao tipo de artigo, houve predomínio de artigos de pesquisa $(\mathrm{N}=72)$ em relação aos artigos teóricos $(\mathrm{N}=7)$. Esse resultado expressa que, 
de um modo geral, os pesquisadores elegem técnicas que apresentam alguma fundamentação estatística para realizar sua avaliação, o que pode gerar resultados mais confiáveis, justificando a pouca prevalência de estudos teóricos em Avaliação Psicológica.

A maioria dos estudos é da área da Psicologia e a temática mais estudada se refere a aspectos psicológicos, corroborando os dados de Sampaio, Sabadini e Linguanotto (2002), Santos, Oliveira e Joly (2003) e Noronha, Vendramini e Freitas (2004). O discurso evidenciou que nem todos os resumos apresentaram o objetivo da pesquisa, dado surpreendente, dada a importância de um artigo científico ter objetivos bem definidos.

Considerando que os resultados apareceram descritos na maioria das publicações $(\mathrm{N}=79)$, a discussão foi descrita em apenas 47 dos 79 resumos. Por sua vez, a metodologia foi descrita em 71 resumos de pesquisa de campo, bem como procedimentos em 65 resumos, evidenciando que houve a exposição clara da metodologia utilizada em quase todos os resumos. Tendo por referência a perspectiva da metaciência (Witter, 1999; 2002) e do conteúdo mínimo necessário para a divulgação científica, ainda há a escassez de informação da área (Poblácion, Witter \& Silva, 2006).

Quanto ao tipo de pesquisa e a análise de dados, respectivamente, foi predominante a pesquisa quantitativa $(\mathrm{N}=64)$. Em sete resumos não foi especificado se o estudo era quantitativo ou qualitativo, e foram identificadas oito pesquisas qualitativas.

Por sua vez, os artigos teóricos foram minoria $(n=3)$ e publicados em revistas de classificação $B 1(n=2)$ e B4 (n=1) na base Lilacs. Todos os resumos analisados descreveram sobre a temática, e a maioria apresentou desenvolvimento e conclusão o que demonstra, que de uma maneira geral, houve a apresentação das informações relevantes ao discurso científico na maioria dos resumos analisados. Tais resultados são semelhantes aos encontrados na análise dos resumos referentes a artigos de pesquisa, cabendo, pois, as mesmas considerações anteriormente já postas.

Em suma, estas informações são importantes a medida que demonstram o que tem sido desenvolvido e pesquisado sobre o tema, bem como revelam que é necessário aprimorar o conhecimento disponível propiciando ao pesquisador referência para definir futuros temas e construtos a serem investigados (Witter, 2006; Lopez Lopez \& cols., 2010; Poblácion, Witter \& Silva, 2006; Silva \& Bianchi, 2001; Witter, 1999, 2002).

Os estudos aqui apresentados e os resultados alcançados assinalam que os estudos transculturais estão em constante expansão e faz-se necessário, portanto, incentivos para investigações dessa natureza procedentes das agências de fomento. Salienta-se ainda, a necessidade de maior rigor por parte das revistas no que se refere à falta de objetivos e discussões nos resumos publicados e a necessidade de normatização dos mesmos, dada a constatação, na análise realizada no presente artigo, sobre a falta de informações fundamentais ao discurso científico. Pesquisas desse porte são relevantes por serem um indicativo das lacunas que precisam ser preenchidas por futuras pesquisas, contribuindo para a disseminação e o progresso do conhecimento produzido institucionalmente nas academias.

\section{REFERÊNCIAS}

Anastasi, A., \& Urbina, S. (2000). Testagem Psicológica. Porto Alegre: Artes Médicas.

Andrade, L. H. S. G., \& Gorenstein, C. (1998). Aspectos gerais das escalas de avaliação de ansiedade. Revista de Psiquiatria Clínica, 25(6), 285-290.

Argollo, N., Bueno, O. F. A., Shayer, B., Godinho, K., Abreu, K., Durán, P., Assis, A., Lima, F., Silva, T. Guimarães, J.,Carvalho, R., Moura, I., \& Capovilla, A. G. S. (2009). Adaptação transcultural da Bateria NEPSY - avaliação neuropsicológica do desenvolvimento: estudo-piloto. Avaliação Psicológica, 8(1), 69-75.

Baquero, G. (1974). Testes psicométricos e projetivos: Medidas psicoeducacionais. São Paulo, SP: Edições Loyola.

Bufrem, L., \& Prates, Y. (2005). O saber científico registrado e as práticas de mensuração da informação. Ciência da Informação, 34(2), 9-25.

Conselho Federal de Psicologia (2001). Resolução n0 25/2001. Recuperado em 23 out. 2010. Disponível em: http://www. pol.org.br.

Gouveia, V. V. (2009). A Avaliação Psicológica no Brasil: caminhos, desafios e possibilidades. Psicologia em foco, 2(1), 110-119.

Joly, M. C. R. A., Martins, R. X., Abreu, M. C., Souza, P. R. R.., \& Cozza, H. F. P. (2004). Análise da Produção Científica em Avaliação Psicológica Informatizada. Avaliação Psicológica, 3(2),121-129.

Joly, M.C.R.A., \& Noronha, A.P.P. (2006). Reflexões sobre a construção de instrumentos psicológicos informatizados. Em A. P. P. Noronha, A. A. A. dos Santos, \& F. F. Sisto (Orgs.), Facetas do fazer em avaliação psicológica. (pp.95105). São Paulo, SP: Vetor Editora Psicopedagógica.

Lopez Lopez, W., Silva, L. M., Garcia-Cepero, M. C., AguilarBustamante, M. C., \& Lopez, E. A. (2010). Panorama general de la producción acadêmica en La Psicologia Colombiana indexada en PsicoRedalyc, 2005-2007. Acta Colombiana de Psicologia,13(2), 35-46.

Maluf, M. R. (2004). A participação de psicólogos brasileiros na Sociedade Interamericana de Psicologia: Contribuições e perspectivas. Interamerican Journal of Psychology, 38(2), 322-332. 
Mattos, P., Segenreich, D., Saboya, E., Louzã, M., Dias, G., \& Romano, M. (2006). Adaptação transcultural para o português da escala Adult Self-Report Scale para avaliação do transtorno de déficit de atenção/hiperatividade (TDAH) em adultos. Revista de Psiquiatria Clínica. [online]. 33(4),188-194. Recuperado em 22 out. 2010. Disponível em: http://www.scielo.br/pdf/rpc/v33n4/a03v33n4.pdf

Nardi, E. A. (1998). Comentário do debatedor: Escalas de avaliação da ansiedade. Revista de Psiquiatria Clínica. [online]. 25(6), 177-183. Recuperado em 22 out. 2010. Disponível em: http://www.hcnet.usp.br/ipq/revista

Noronha, A. P. P. (2002). Os problemas mais graves e mais freqüentes no uso dos teste psicológicos. Psicologia: Reflexão e Crítica, 15(1), 135-142.

Noronha, A.P.P., \& Baptista, M.N. (2007). Relação entre metodologia e avaliação psicológica. Em M. N. Baptista, \& D. C. De Campos. Metodologia de pesquisa em ciências: análise quantitativa e qualitativa. (pp. 49-60). Rio de Janeiro, RJ:LTC.

Noronha, A. P. P., Sartori, F. A., Freitas, F. A. \& Ottati, F. (2001). Informações contidas nos manuais de testes de inteligência publicados no Brasil. Psicologia em Estudo, 6(2), 101-106.

Noronha, A. P. P., Vendramini, C. M. M., Canguçu, C., Souza, C. V. R., Cobêro, C., de Paula, L. M., Franco, M. de O., Lima, O. M. P., Guerra, P. B. de C., \& Filizatti, R. (2003). Propriedades psicométricas apresentadas em manuais de testes de inteligência. Psicologia em Estudo. 8(1), 93-99.

Noronha, A. P., Vendramini, C. M. M., \& Freitas, F. A. (2004). A avaliação psicológica no I Congresso Brasileiro de Psicologia. Em C. Machado, L. S. Almeida, M. Gonçalves \& V. Ramalho (Orgs.), Avaliação Psicológica: formas e contextos (pp. 11-15). Braga: Psiquilíbrios Edições.

Oliveira, K. L., Noronha, A. P. P., Dantas, M. A. \& Santarém, E. M. S. (2005). O psicólogo comportamental e a utilização de técnicas e instrumentos psicológicos. Psicologia em Estudo, 10, 127-135.

Pacheco, E. M. C. (2005). Produção científica e avaliação psicológica. Em G. P. Witter, (Org.). Metaciência e Psicologia. (pp. 7-33) Campinas, SP: Editora Alínea.

Packer, A., \& Meneghini, R. (2006). Visibilidade da produção cientifica. Em: D. A.Poblacion,(org). Comunicação \& produção científica: contexto, indicadores avaliação. (pp. 237-259) São Paulo,SP: Angellara.

Paixao Jr, C. M., Reichenheim, M. E., Moraes, C. L., Coutinho, E. S. F., \& Veras, R. P. (2007). Adaptação transcultural para o Brasil do instrumento Caregiver Abuse Screen (CASE) para detecção de violência de cuidadores contra idosos. Cadernos de Saúde Pública, 23(9), 2013-2022.

Pasquali, L. (1997). Psicometria: Teoria e aplicações. Brasília, DF: Editora da UnB.
Pasquali, L. (1998). Princípios de elaboração de escalas psicológicas. Revista de Psiquiatria Clínica, 25(5), 206-21.

Pasquali, L. (1999). Instrumentos psicológicos: Manual prático de elaboração. Brasília,DF: LabPAM/IBAPP.

Pesce, R. P., Assis, S.G., Avanci, J. Q., Santos, N. C., Malaquias, J. V., \& Carvalhaes, R. (2005). Adaptação transcultural, confiabilidade e validade da escala de resiliência. Cadernos de Saúde Pública, 17(4), 887-896.

Poblácion, D. A., Witter, G. P., \& Silva, J. F. M.(2006). Comunicação \& produção científica: contexto, indicadores e avaliação. São Paulo,SP: Angellara Editora.

Primi, r. (2010) Avaliação Psicológica no Brasil: Fundamentos, situação atual e direções para o futuro. Psicologia: Teoria e Pesquisa, 26, 25-35.

Ribas Jr, R. de C., Portugal, C. M., Pitrowsky, L. T., Cunha, M. P. da, Blanco, P. R., Saliba, V. A., Chaves Júnior. I. P., Faria, C. D. C. de M., \& Teixeira-Salmela, L. F.(2008). Propiedades Psicométricas da Motor Activity Log: uma revisão sistemática da literatura. Fisioterapia Movimento, 21(3), 59-67.

Saliba, V. A., Magalhães, L. de C, Faria, C. D. C. de M., Laurentino, G. E. C. , Cassiano, J. G., \& TeixeiraSalmela, L. F.. (2011). Adaptação transcultural e análise das propriedades psicométricas da versão brasileira do instrumento Motor Activity Log. Revista Panamericana de Salud Pública, 30(3), 262-271. Acessada em 16/9/2014: http://www.scielosp.org/scielo.php?script=sci arttext\&pid=S1020-49892011000900011\&lng=en\&tlng= pt. $10.1590 / \mathrm{S} 1020-49892011000900011$

Sampaio, M. I. C., Sabadini, A. A. Z. P., \& Linguanotto, A. R. J. (2002). Periódicos científicos: características e exigências. Mudanças: Psicologia da Saúde, 10(1), 184-200.

Santos, A. A. A., Oliveira, K. L., \& Joly, M. C. A. (2003). Produção científica da área de escolar no I Congresso Brasileiro de Psicologia Ciência e Profissão. Psicologia Escolar e Educacional, 7(2), 135-144.

Silva, J. A., \& Bianchi, M. de L. P. (2001). Cientometria: A métrica da ciência.Paidéia, 11(20), 5-10.

Silva, F. C. Da, \& Thuler, L. C. S. (2008). Tradução e adaptação transcultural de duas escalas para avaliação da dor em crianças e adolescentes. Jornal de Pediatria, 84(4), 344-349.

Sisto, F. F. (2004). Precisão. Universidade São Francisco, Itatiba. [manuscrito não publicado].

Tesch, F. C., Oliveira, B. H. de, \& Leão, A. (2008) Equivalência semântica da versão em português do instrumento Early Childhood Oral Health Impact Scale. Cadernos de Saúde Pública, 24(8), 1897-1909.

Urbina, S. (2007). Fundamentos da testagem psicológica. Porto Alegre, RS. Artmed. 
Van Kolck, O.L. (1974). Técnicas de exame psicológico e suas aplicações no Brasil. Petrópolis, RJ: Vozes.

Witter, G. P. (1999). Metaciência e leitura. Em G. P. Witter (Org.). Leitura: Textos e pesquisas. (pp. 13 -22). Campinas, SP: Editora Alínea.
Witter, G. P. (2002). Produção científica sobre Estresse e Prevenção. Em: G. P Witter. (Org.) Psicologia: Tópicos Gerais. (pp. 213-228). Campinas, SP: Editora Alínea.

Witter, G.P. (2006). Envelhecimento: referenciais teóricos e pesquisas. Campinas, SP: Editora Alínea. 